Estudios sobre el Mensaje Periodístico

ISSN-e: $1988-2696$

http://dx.doi.org/10.5209/ESMP.55619

\title{
El tratamiento periodístico sobre la corrupción política. Análisis comparado del caso SQM en Chile y del caso de Bárcenas en España
}

\author{
Salomé Sola-Morales ${ }^{1}$; Ricardo Rivera Gallardo²
}

Recibido: 19 de marzo de 2016 / Aceptado: 5 de septiembre de 2016

Resumen. Este artículo empírico analiza cualitativamente el tratamiento periodístico de la corrupción política en España y en Chile. Concretamente, se centra en el caso chileno "Soquimich" y en el caso español "Bárcenas". Para ello, se compara el discurso noticioso de los diarios chilenos El Mercurio y La Tercera y de los periódicos españoles El País y El Mundo en relación a los siguientes ejes: 1) Rol de los personajes y caracterización de los mismos y 2) Estrategias discursivas subyacentes en el discurso mediático. Si bien existen diferencias en la cobertura noticiosa en ambos países, los resultados obtenidos permiten concluir que el tratamiento de la corrupción es simplificado e incompleto. Eso se debe a que los relatos analizados se focalizan en los "corruptos" sin cuestionar las verdaderas raíces de la corrupción.

Palabras clave: Corrupción; medios de comunicación; discurso; España; Chile.

\section{[en] The Journalist Discourse on Political Corruption. A Comparative Analysis of the SQM case in Chile and the Bárcenas case in Spain}

\begin{abstract}
This empirical article analyzes qualitatively press coverage of political corruption in Spain and Chile. Specifically, the study focuses on the Chilean case "Soquimich" and in the Spanish case "Barcenas". For this purpose, the news discourse of Chilean daily El Mercurio and La Tercera and Spanish newspapers El País and El Mundo were compared regarding the following axes: 1) Role of the characters and characterization of them and 2) Discursive strategies underlayed by the media discourse. While there are differences in news coverage of corruption in both countries, the results obtained indicate that treatment of corruption is simplified and incomplete. That is because the stories analyzed are focused on corrupt - the particular characters - without questioning the real roots of corruption.
\end{abstract}

Keywords: Corruption; media; discourse; Spain; Chile.

Sumario. 1. Introducción; 1.1. Contexto chileno y español; 1.2. Los casos analizados; 1.2.1. Chile, el caso SQM; 1.2.2. España, el caso Bárcenas. 2. Objetivos e hipótesis. 3. Fuentes y metodología. 4. Resultados; 4.1. Rol de los personajes y su caracterización; 4.1.1. España "Caso Bárcenas"; 4.1.2. Chile "Caso SQM"; 4.2. Estrategias discursivas. 5. Discusión. 6. Conclusiones. 7. Referencias bibliográficas.

Cómo citar: Sola-Morales, Salomé y Rivera Gallardo, Ricardo (2017): “El tratamiento periodístico sobre la corrupción política. Análisis comparado del caso SQM en Chile y del caso de Bárcenas en España”, en Estudios sobre el Mensaje Periodístico 23 (1), 647-662.

\footnotetext{
1 Universidad de Santiago de Chile.

E-mail: ssolamorales@gmail.com

2 Universidad Autónoma de Barcelona.

E-mail: ricardo.rivera.gallardo@gmail.com
} 


\section{Introducción}

La corrupción política no se trata de un fenómeno novedoso ni exclusivo de sociedad alguna (Pérez Tapias, 1995). De hecho, se encuentra presente en numerosos ámbitos de la vida pública (González, 1999) y, a lo largo de la historia, ha afectado a la administración pública y a los cargos electivos del Estado (Weber, 1922; Queralt, 2012). Esto ha ocurrido independientemente del tipo de gobierno, ya sea autoritario o democrático (González, 1999), o de su forma de organización o ideología. Es más, se pudieron apreciar casos tanto en EEUU como en la URSS (Hobsbawm, 1999; Fontana, 2011). Pero además, la corrupción también se puede dar en el mundo privado (Hodgson y Jiang, 2008), en especial cuando las entidades privadas estafan a las instituciones fiscalizadoras de los aparatos públicos (Queralt, 2012).

Existe una amplia literatura científica acerca del concepto de corrupción desde enfoques epistemológicos diversos tales como la economía, la antropología, la ciencia política o la jurídica. No obstante, algunos investigadores afirman que se trata de una noción difusa rodeada de un vacío conceptual (Hodgson y Jiang, 2008). Otros han realizado tipologías de corrupción refiriéndose al nivel que puede alcanzar - político, económico, administrativo- (Boehm y Graf, 2009) o han demarcado diferencias clave respecto a otros conceptos tales como "clientelismo político" (Caciagli, 1996; Corzo, 2002; Rehren, 2000).

Cuando hablamos de corrupción política es preciso hacer alusión al marco en que se produce la ilegalidad y al nivel alcanzado: se puede tratar de corrupción interpersonal, es decir, de delitos provinciales o de corrupción de alto nivel, es decir, de delitos cometidos por los poderes del Estado (Boehm y Graf, 2009). Esta perspectiva está muy relacionada con los procesos que vive cada nación (Gumucio, 2005).

El fenómeno de la corrupción, no solamente está relacionado con los países menos desarrollados, sino que también se da en los países con más nivel de crecimiento (Bautista, 2007). Según Bautista, desde los años 90 se viene dando, además, una mayor cantidad de casos y un aumento de la visualización de los mismos. Esta situación se ha dado, en parte, gracias al creciente rol fiscalizador de los medios de comunicación y al surgimiento de una serie de instituciones nacionales e internacionales que miden el grado de corrupción por países como la agencia Transparencia Internacional.

Las causas de la corrupción son múltiples y dependiendo del contexto de cada país tendrán una expresión particular. Para Kauffman (2000), la corrupción puede ser generada por distintas causales: el nivel de desarrollo del país; la diferencia entre los salarios de las instituciones públicas o privadas; las políticas proteccionistas y el deficiente desempeño del mercado financiero; la existencia de instituciones de vigilancia para detectar los casos de corrupción; la ausencia de libertades en un país y, por último, la existencia de un poder judicial débil.

En las actuales sociedades democráticas la corrupción se presenta como una anomalía peligrosa o un "cáncer mortal" que corroe la institucionalidad y a los sujetos (Pérez Tapias, 1995). Los efectos de la corrupción van mucho más allá del simple malestar de la ciudadanía y terminan deteriorando la democracia (Barreiro y Sánchez-Cuenca, 2000). Durante las elecciones, por ejemplo, la corrupción provoca un clima de inestabilidad o de apatía política que hace que el foco de las discusiones y de los programas de gobierno se vean reducidos a un segundo plano. 
Además el descrédito de los partidos, que caen en actos de corrupción, pone en peligro la participación, de igual manera. (Pérez y López, 2014; Ordóñez Pérez, 2012). En este contexto, la ciudadanía tiende a asumir que la corrupción es un fenómeno global y generalizado, lo cual genera un desinterés por la política (Laguna Platero, 2013). Es más, la percepción de que la corrupción crece y de que los gobiernos no toman las medidas necesarias para solucionar el problema, ha ido aumentando (Muñoz, 2012). Así, los ciudadanos tienden a distanciarse de "los políticos" y generan un "otro" alejado del "nosotros". Pero además cuando la ciudadanía está desinformada políticamente y es poco participativa, la corrupción se desarrolla con mayor facilidad. Esto da pie a que la moralidad democrática pierda peso y se configuren formas de totalitarismo (Hodgson y Jiang, 2008).

La corrupción también tiene consecuencias en la economía. Así los estados que sufren de desregulación y de tasas de corrupción altas tienden a la inestabilidad, puesto que la ausencia de normas legales infringe severos daños al mercado (Hodgson y Jiang, 2008). En definitiva, el impacto de la corrupción es muy negativo ya que tiene un efecto directo sobre el índice de desarrollo humano (Akçai, 2006; Quizilbash, 2001), sobre el crecimiento económico de un país (Mauro, 2002). Además, genera un aumento de la desigualdad de ingresos, mayor abandono escolar o menor inversión directa extranjera, por citar algunas de las consecuencias más relevantes (Larrú, 2009).

En este contexto, el rol de los medios es clave. Primero porque la función de los mismos debería ser la de fiscalizar. De hecho, algunos autores han subrayado que las noticias sobre corrupción son un indicador de calidad de la democracia, en contraposición con las dictaduras (Castells, 2003), ya que "la libre expresión en los medios es el único freno posible a la arbitrariedad de los que gobiernan" (Martínez Gallego, 2013, p. 100). Segundo, porque "el conocimiento público del escándalo por corrupción y los juicios que la sociedad formula a su respecto están mediatizados" (Martínez Gallego, 2013, p. 104). Esto se debe a que otorgan un carácter simbólico a las narraciones sobre corrupción y a la repercusión social de la misma, mediante la cobertura de la acusación o la reacción del acusado (Canel y Sanders, 2005, 2006). Tercero, porque los medios deberían responder a una "moral democrática" (Pérez Tapias, 1995) ofreciendo las claves de lo que es un sistema sano políticamente. Sin embargo, en ocasiones, los medios dotan de un carácter dramático a las noticias mediante la "espectacularización" de los casos de corrupción (Martínez Gallego, 2013), empujados por su estructura mercantilista. Justamente en este sentido la corrupción puede ser entendida como un fenómeno de corte interpretativo. Esto se debe a que los mass media han contribuido a formar una teoría de la corrupción y han formado una interpretación del significado de la misma (Hierro, 2014; Martínez, 2013; Zamora y Marín, 2010, 2011).

Con el fin de conocer cuál es el discurso mediático que se trasmite a las audiencias acerca de esta lacra que parece asolar todos los sistemas políticos democráticos en menor o mayor medida, este artículo analizará cuál es el tratamiento periodístico de la corrupción política en España y en Chile. Concretamente, el estudio se centrará en el caso chileno Soquimich y en el caso español Bárcenas. Para ello, se comparará el discurso noticioso de los diarios chilenos El Mercurio y La Tercera y de los periódicos españoles El País y El Mundo en relación a los siguientes ejes: 1) Rol de los personajes y caracterización de los mismos y 2) Estrategias discursivas subyacentes en el discurso mediático a partir del enfoque determinado por el medio. 


\subsection{Contexto chileno y español}

Los casos de corrupción han sido una constante en la historia de Chile. La condición estructural o ideológica de la corrupción, que se desarrolló desde mediados y finales de la dictadura militar (Orellana, 2007), permite indicar que Chile sufre de un constante proceso de corrupción. El fenómeno de privatización irregular a finales de la dictadura militar y la expansión de las empresas durante los gobierno de la Concertación representan un hecho de corrupción en sí mismo (Mönckeberg, 2002). Es más, la influencia de las grandes empresas y el proceso de separación de la ética y la política son constantes en el tiempo (Rehren, 1996, 2000; Gumucio, 2005).

Sin embargo, según el "Índice de Percepción de la Corrupción" (Corruption Perceptions Index, CPI), de 2015, entregado por la agencia de "Transparencia Internacional", Chile se encuentra entre los países con un menor índice de percepción en casos de corrupción, ubicándose en el puesto número 23 a nivel mundial. Además, Chile es considerado el segundo país con menor corrupción en América Latina, quedando detrás de Uruguay.

En contraste con estos datos, durante la última década han aparecido una serie de escándalos políticos en el país que ponen en cuestión dichos indicadores y han convertido la corrupción en unos de los leitmotiv de las agendas noticiosas. Ya desde 2008 se podía apreciar un alza en el nivel de desconfianza de la ciudadanía hacia la clase política (Luna, 2008). Es más, entre los años 1990 y 2008 los escándalos por corrupción llegaron a ser 15, de los cuales solo 13 fueron cubiertos por la prensa escrita (Balán, 2009). Durante 2014 y, especialmente, en 2015 una serie de políticos se han visto involucrados en los casos: Caval, SQM, PENTA, Corpesca, por financiamiento ilegal y malversación de dineros municipales. Además, datos de la encuesta del Centro de Estudios Públicos (CEP), de noviembre del 2015, señalaron que la corrupción es la quinta preocupación más relevante para los encuestados, superando al empleo, la pobreza y la desigualdad.

Los medios de comunicación han sido fundamentales para fiscalizar los procesos de corrupción en Chile y en el conjunto América Latina (López, 2002; Morales, 2006). En el actual escenario, no solo el sistema de justicia es quien trabajaba en búsqueda de las evidencias, sino también la prensa (Kaufmann, 2000). Por otra parte, el hecho de que los medios puedan generar opinión pública sobre los casos de corrupción, presenta una presión concreta para los actores que están presentes en el caso (Zamora y Marín, 2011). Sin embargo, según los datos entregados por el Barómetro de Corrupción Global, un $48 \%$ de los chilenos piensa que los medios de comunicación también son corruptos, lo que pone de manifiesto la importancia que tiene la prensa en el momento de focalizar y hablar de una noticia.

Según los indicadores de percepción de corrupción, España se mantiene de forma estable desde la década del 2000 en una buena posición. Concretamente, en el año 2015, ocupó la posición 36 del ranking de los países con menor CPI. Sin embargo, con los casos más recientes de corrupción, el fenómeno ha pasado a situarse en el centro del debate público, lo cual haría cuestionar los resultados de este ranking. De hecho, en el "Barómetro de Octubre 2015" del Centro de Investigaciones Sociológicas (CIS), el problema de la corrupción se encuentra situado como el segundo problema más importante para la sociedad española. De hecho un $83 \%$ de los encuestados cree que los partidos políticos son corruptos, un $67 \%$ cree 
que el parlamento español es corrupto y un $51 \%$ piensa lo mismo del sistema de justicia español. Por último, señalar que para un $41 \%$ de los españoles la prensa es corrupta, poniendo de manifiesto una situación muy similar a la de Chile.

A pesar de que los resultados mostrados por estos índices evidencian el alto nivel de corrupción y percepción negativa de la misma tanto en Chile como en España, algunos investigadores han advertido que estos datos no son precisos (Kauffman, 2000 , p. 369). Es más, la medición de la corrupción trae consigo problemas metodológicos inherentes (Villorria y Jiménez, 2012), que impiden concluir acerca del grado de corrupción de un país y el consecuente desgaste de la ciudadanía.

\subsection{Los casos analizados}

\subsubsection{Chile, el caso SQM}

La empresa minera "Sociedad Química y Minera de Chile" (SQM) se ha visto involucrada en casos de corrupción desde el año 2012. No obstante, ya desde su privatización (1983-1988) se denunciaron irregularidades. Es más, el mismo proceso de privatización fue considerado como irregular ya que Julio Ponce Lerou se apropió en 1980 de forma indebida de una empresa estatal, siendo además yerno del dictador Augusto Pinochet (Mönckeberg, 2002).

El proceso comenzó a tener un seguimiento en los medios de comunicación desde el año 2012, por una estafa realizada por los dueños de SQM. Julio Ponce Lerou había generado un holding que iba en detrimento del resto de los accionistas. Así las primeras denuncias fueron realizadas por los accionistas minoritarios que observaron que la empresa realizaba negocios que solo beneficiaban a las empresas mayoritarias de Ponce Lerou. Pero el caso de corrupción se presentó con más fuerza en los medios de comunicación cuando se destapó que la empresa eludía pagar impuestos y financiaba ilegalmente a partidos políticos y, en especial, a representantes o candidatos a cargos públicos.

\subsubsection{España, el caso Bárcenas}

El caso Bárcenas se ha transformado en el principal acontecimiento de corrupción que han cubierto los medios de comunicación en España en las últimas décadas. Desde principios del año 2013, cuando se descubrió el pago de sobresueldos a representantes del Partido Popular, el tema ha ocupado las agendas mediáticas. El proceso judicial que hasta la fecha sigue en trámite, ha significado una trama compleja, en la cual el Gobierno de Mariano Rajoy se ha visto implicado (Ekaizer, 2013).

Con respecto al caso Bárcenas, hay que mencionar que existe un relato y un proceso, que se inicia gracias a la cobertura realizada por los medios de comunicación. La denuncias realizadas por los periódicos El Mundo y El País fueron las que destaparon los sobresueldos que entregaba Luis Bárcenas a militantes del PP de manera ilegal. El ex-tesorero, que trabajó desde 1990 hasta el 2009 y estuvo a cargo de la gestión económica durante el gobierno de José María Aznar, era el encargado de reunir el financiamiento de las grandes empresas y traspasarlas al partido. 


\section{Objetivos e hipótesis}

El objetivo general de esta investigación es analizar y comparar cuál es el tratamiento mediático de la corrupción política en España y en Chile, a través de los casos SQM y Bárcenas en los diarios chilenos El Mercurio y La Tercera, y los españoles, El País y El Mundo. Los objetivos específicos son:

- Conocer cuál es el rol de los personajes en el discurso mediático de los dos casos y cuál es la categorización que los medios hacen de cada uno de ellos.

- Analizar cuáles son las estrategias discursivas utilizadas por los medios en el tratamiento de ambos casos.

Las hipótesis planteadas en esta investigación son las siguientes:

H1: El discurso mediático tanto en Chile como en España se centra en los sujetos corruptos (los culpables o villanos) y no en los corruptores (el sistema o los partidos políticos), debilitando así responsabilidades y de los grupos financieros y políticos y culpabilizando a sujetos individuales.

H2: La cobertura informativa de los casos Bárcenas y SQM se caracteriza por una tendencia a la "omisión" de elementos clave en el discurso de la corrupción.

\section{Fuentes y metodología}

Con el fin de abordar y comparar empíricamente la representación simbólica de la corrupción política y la cobertura periodística de la misma en Chile y España, se ha realizado un análisis de contenido, de tenor cualitativo, de los dos diarios con mayor tirada de cada país.

La muestra la componen un total de 321 noticias sobre el caso Bárcenas y el caso SQM. En el caso de España se han analizado todas las noticias publicadas en los periódicos El Mundo y El País (n=177). En el caso chileno se han analizado todas las noticias publicadas en La Tercera y El Mercurio $(\mathrm{n}=144)$. Para poder realizar la comparativa entre ambos países, y dado que los casos tienen desarrollos temporales asincrónicos, se han escogido los primeros meses del estallido mediático de ambos casos. En el caso español se escogieron los meses de enero y febrero de 2013 y en el caso chileno febrero, marzo y abril de 2015. El análisis de contenido fue realizado en torno a dos ejes:

1) Rol de los personajes y la caracterización de los mismos se analizó en base a las siguientes categorías: corrupto o corruptor; víctima, héroe o villano. En el caso español se seleccionaron los 10 personajes clave del caso. Concretamente se delimitaron cinco personajes concretos: Bárcenas, PP, el juez Ruz, Mariano Rajoy, Cospedal. Estos personajes concretos de la noticias fueron seleccionados según la mención que se realizaban a estos para poder describir el enfoque que tienen los medios de comunicación. Por otro lado, cinco personajes abstractos: sistema (entendido como el conjunto de leyes y relaciones entre instituciones público-privadas), gobierno (el poder ejecutivo), empresas (sector privado), sociedad (la ciudadanía) y partidos políticos. El análisis de 
personajes abstractos nos permite identificar las omisiones y los énfasis que busca realizar el medio con las estructuras en las que se desenvuelven los personajes concretos. En el caso chileno, a su vez, se delimitaron 10 personajes clave. Se delimitaron cinco personajes concretos: Julio Ponce Lerou, Soquimich, Sabaschahuán, Servicio de Impuestos Internos, Nueva Mayoría y Alianza por Chile, y cinco personajes abstractos: sistema, gobierno, empresa, sociedad, partidos. En primer lugar, a cada uno de los ítems seleccionados se le asignaron cuatro roles: corrupto, corruptor, otro rol y no mencionado. El valor "corrupto" corresponde al sujeto o institución que comete el acto de corrupción. El valor "corruptor" representa a los que corrompen al corruptor. "Otro rol" designa casos diferentes al propuesto y "no mencionado" hace alusión a la negación de todos los anteriores. En segundo lugar, se determinó si cada uno de los personajes analizados en ambos casos eran representados como víctimas, héroes o villanos. Este análisis se realizó por medio de la configuración del discurso de dichos medios sobre los personajes.

2) Estrategias discursivas subyacentes en la noticia. En este caso se delimitaron cuatro estrategias retóricas clave con el fin de evidenciar cuál es el tratamiento que los medios dan a la corrupción. Las categorías fueron: a) mitigación, entendida como una atenuación de la fuerza ilocucionaria; b) idealización, referida a la intensificación o sobrevaloración positiva de los atributos de algún personaje; c) omisión, determinada ante la ausencia de información relevante y necesaria para la comprensión de la noticia; y d) polarización, efectuada a través de las estrategias de presentación positiva de "nosotros" y de presentación negativa de los "otros". Una vez realizado el análisis, la información fue explotada con el programa estadístico SPSS, con el fin de obtener una valoración descriptiva de los resultados mediante un análisis de frecuencias simples.

\section{Resultados}

\subsection{Rol de los personajes y su caracterización}

\subsubsection{España "Caso Bárcenas"}

A la luz de los resultados derivados del análisis de los personajes del Caso Bárcenas se pudo inferir que el discurso periodístico de El País y El Mundo describe como corruptos a cinco personajes principalmente de los 10 analizados. En el gráfico $\mathrm{N}^{\circ} 1$ se puede observar el porcentaje del rol de los personajes y la caracterización.

En primer lugar, el Partido Popular (PP) es representado en las noticias como una institución corrupta en el 80,8\%, se le relaciona directamente con Bárcenas. En segundo lugar, el ex-tesorero del PP, Luis Bárcenas, es catalogado en ambos medios como corrupto en un $96,0 \%$ de las noticias analizadas, por ende, el enfoque de la noticia se construyó en relación al encargado de las finanzas. En tercer lugar, el juez Ruz, encargado de llevar el caso Bárcenas, es poco mencionado, y en el $24,9 \%$ de las menciones que se hacen al juez, no tenía relación con el valor de corrupto y corruptor. En cuarto lugar, el presidente Mariano Rajoy es catalogado como corrupto en un $31,1 \%$ de las noticias analizadas, sin embargo, en el 55,9\% de los artículos 
estudiados, el presidente no es mencionado. En quinto lugar, la encargada del PP, Cospedal, no es mencionada prácticamente, en el $75,1 \%$ de los textos estudiados es dejada de lado.

Con respecto a las instituciones más abstractas se puede ver que el mensaje de los medios se centró en aspectos específicos, en especial en los corruptos. En primer lugar, el sistema no es mencionado en un 80,2\%, por lo cual, El País y El Mundo, proyectan un discurso focalizado en personajes particulares. En segundo lugar, el Gobierno es descrito en un 56,5\% como corrupto, siguiendo la línea de las menciones a los personajes concretos. En tercer lugar, las empresas son omitidas en el $87 \%$ de las noticias, lo cual sumado a la omisión que se realiza del sistema disminuye la posibilidad de encontrarse con el corruptor dentro del texto. En cuarto lugar, la sociedad, en el $88,1 \%$ de los casos la sociedad no se presenta dentro de la noticia, por ende, se puede interpretar que está ausente la víctima del delito. En quinto lugar, los partidos políticos, en general (sean del color ideológico que sean), son descritos como corruptos en un $79,7 \%$ de las noticias estudiadas, esto ocurre por un tratamiento mediático con omisiones sobre la investigación.

De hecho, al analizar las variables de sistema, de empresa y de sociedad también se puede destacar que no son mencionadas en la noticia, solo se puede observar que en un $13 \%$ el sistema es relacionado con el valor de "corrupto" y que las empresas que financian los sobresueldos son poco mencionadas por los medios. Los partidos y el gobierno son expuestos como corruptos, estos tiene un $79,7 \%$ y $56,5 \%$ respectivamente, puesto que las noticias entregan la información sin explicaciones o interpretaciones previas que maticen a los sectores del gobierno y a los partidos no implicados.

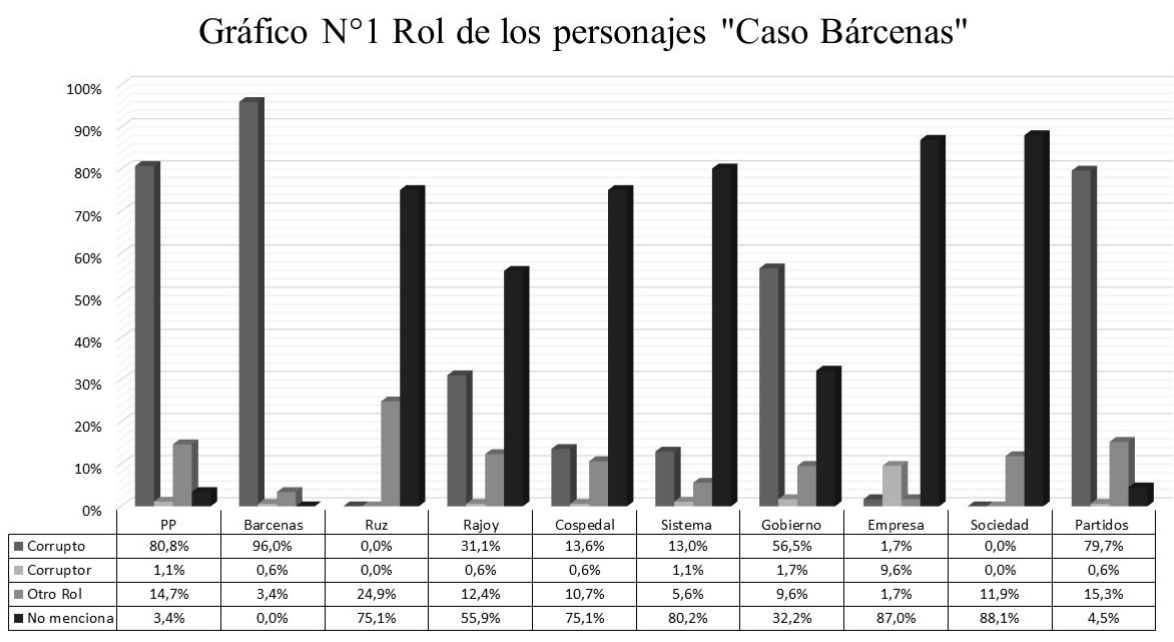

Fuente: Elaboración propia

En el análisis de los personajes, se ocuparon las mismas variables, sin embargo, para describir los elementos narrativos propuestos por los periodistas, indicaremos cuatro valores nuevos. El objetivo de esta categorización es describir la noticia a partir de la valoración personajes, los cuales identificamos con instituciones o sujetos 
claves del "Caso Bárcenas". Por este motivo, ocuparemos los siguientes personajes como valores: el héroe, el villano, la víctima y no mencionado.

En el gráfico $\mathrm{N}^{\circ} 2$ se puede observar como las variables concretas juegan un rol dentro de la narración. En primer lugar, el PP es categorizado como un villano. De hecho, una gran cantidad de periodistas optó por relacionar y valorar al PP como una institución que se benefició de los sobresueldos ilegales, siendo catalogado en un $76,8 \%$ de los artículos como villano. En segundo lugar, se puede observar a Bárcenas, caracterizado como villano, en un $98,9 \%$ de los casos, lo cual permite identificar un culpable directo del caso de corrupción. En tercer lugar, está el juez Ruz, el cual no sale mencionado en $75,1 \%$ de los casos, sin embargo cuando es mencionado, en el $24,3 \%$ es referenciado como el héroe. En cuarto lugar, Mariano Rajoy no es mencionado en un 55,9\% de los casos, y cuando es mencionado, en el $31,6 \%$ es caracterizado como villano. En quinto lugar, María Dolores de Cospedal no es mencionada en la mayoría de las noticias analizadas, en el 75,1\% de los casos, y cuando es caracterizada los es como víctima $(10,7 \%)$ o como villana $(13,6 \%)$.

Los personajes abstractos que se presentan en el gráfico $\mathrm{N}^{\circ} 2$ indican similitudes con los personajes concretos. En primer lugar, el sistema no aparece mayormente mencionado, es más, en el $80,2 \%$ de las noticias se evade la cuestión del sistema. En segundo lugar, el gobierno es presentado mayoritariamente como villano en un $53,1 \%$ de los textos estudiados. En tercer lugar, las empresas que financian a los corruptos no son mencionadas en el $87 \%$ de las noticias analizadas. En cuarto lugar, la sociedad no es mencionada en un $88,1 \%$. En quinto lugar, los partidos políticos son relacionados como causantes de la corrupción en el 70,6\% de los casos y solo en $22 \%$ son indicados como víctimas de la corrupción.

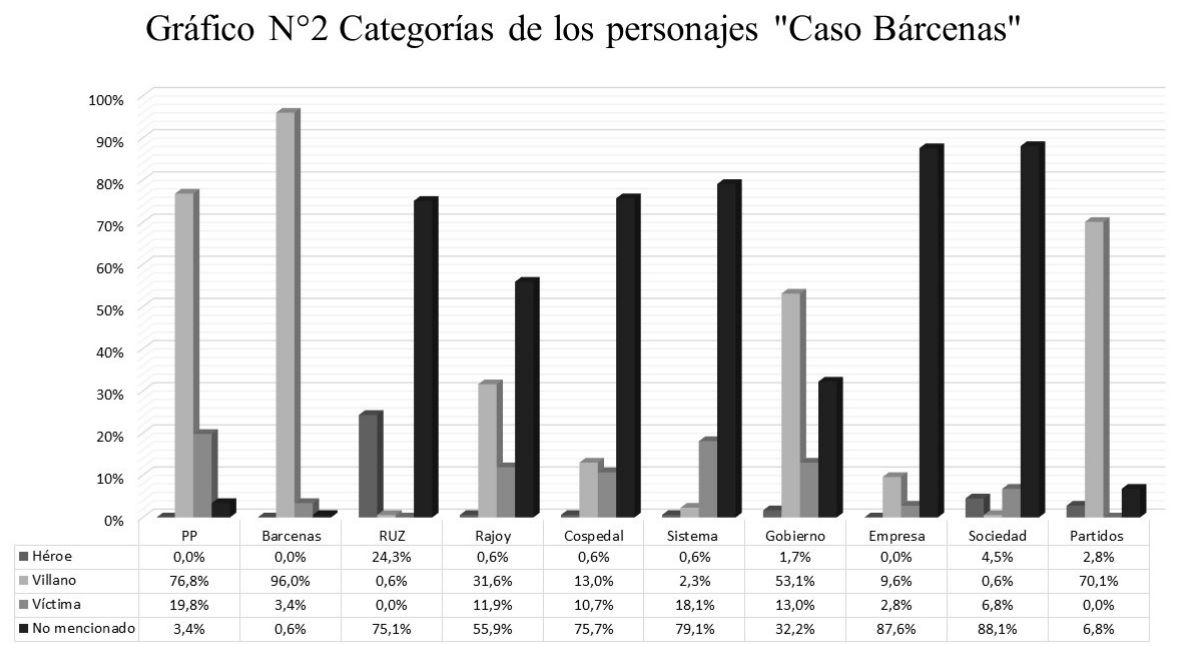

Fuente: Elaboración propia

\subsubsection{Chile "Caso SQM"}

En el gráfico 3 se pueden observar los roles adjudicados a los diferentes personajes. En primer lugar, Julio Ponce Lerou (JPL) no es mencionado en un 72,9\%, sin embargo, en el $26,4 \%$ es presentado como corruptor. En segundo lugar, la empresa SQM 
se identificó directamente como corruptora en el 93.8\%. En tercer lugar, el fiscal nacional, Sabas Chahuán, no es mencionado en la mayoría de los casos (63,9\%). En cuarto lugar, el personaje Servicios de Impuestos Internos (SII) está presente en el $54,2 \%$ con un rol no corrupto. En quinto lugar, las coaliciones de la Nueva Mayoría (NM) y la Alianza son mencionadas como corruptas en el 51,4\% de las noticias.

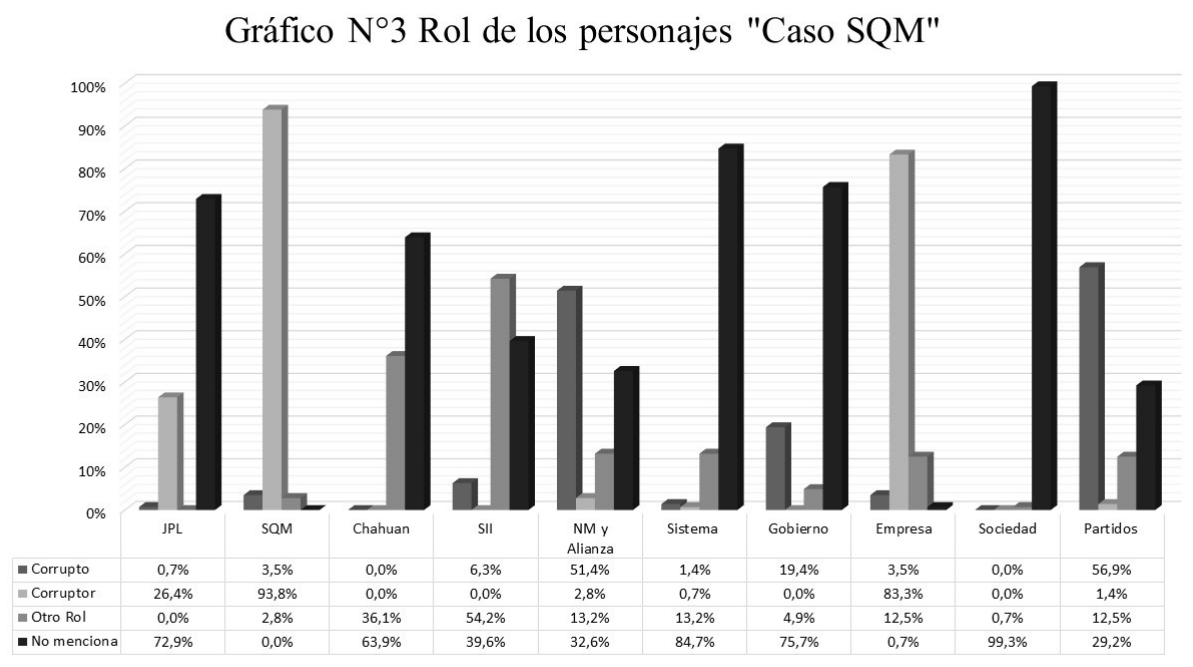

Fuente: Elaboración propia

En cuanto a los personajes abstratos del caso SQM, el sistema no es mencionado en el $84,7 \%$. El gobierno no es mencionado en el $75,7 \%$ de las noticias trabajadas. En tercer lugar, la empresa es señalada en el 83,3\% como corruptor. En cuarto lugar, la sociedad no es mencionada en el 99,3\% de los textos examinados. En quinto lugar, los partidos políticos son tratados en el 56,9\% de las noticias como corruptos.

El trabajo de los periodistas con los personajes del "Caso SQM" marca una diferencia entre las instituciones y los sujetos. La información entregada por El Mercurio y La Tercera, que se puede observar en el gráfico $\mathrm{N}^{\circ} 4$. En primer lugar, Julio Ponce Lerou es catalogado como un corrupto, a pesar de eso, no es mencionado en el 72,9\% de las noticias analizadas. En segundo lugar, la empresa SQM es indicada como el principal villano, un $97,9 \%$ identificaron a la empresa como corruptor. En tercer lugar, Sabas Chahuán no es mencionado en el 63,3\%, pero cuando fue mencionado se le colocó como héroe $(32,6 \%)$. En cuarto lugar, el SII es mencionado como héroe en el $53,5 \%$. En quinto lugar, la NM y la Alianza son presentadas como villanos en el $54,2 \%$, ya que recibieron beneficios de SQM.

En el caso de los personajes abstractos se puede identificar una relación similar a la de las indicaciones de los personajes concretos. El gráfico $\mathrm{N}^{\circ} 4$ indica la relevancia de los corruptores en el proceso informativo. En primer lugar, el sistema no es mencionado (84,7\%). En segundo lugar, el gobierno no es mencionado en un $75,7 \%$. En tercer lugar, la empresa es indicada en el $86,8 \%$ como villano. En cuarto lugar, la sociedad no es mencionada en la casi la totalidad de las noticias estudiadas. En quinto lugar, los partidos son señalados como villanos en el $58,3 \%$. 


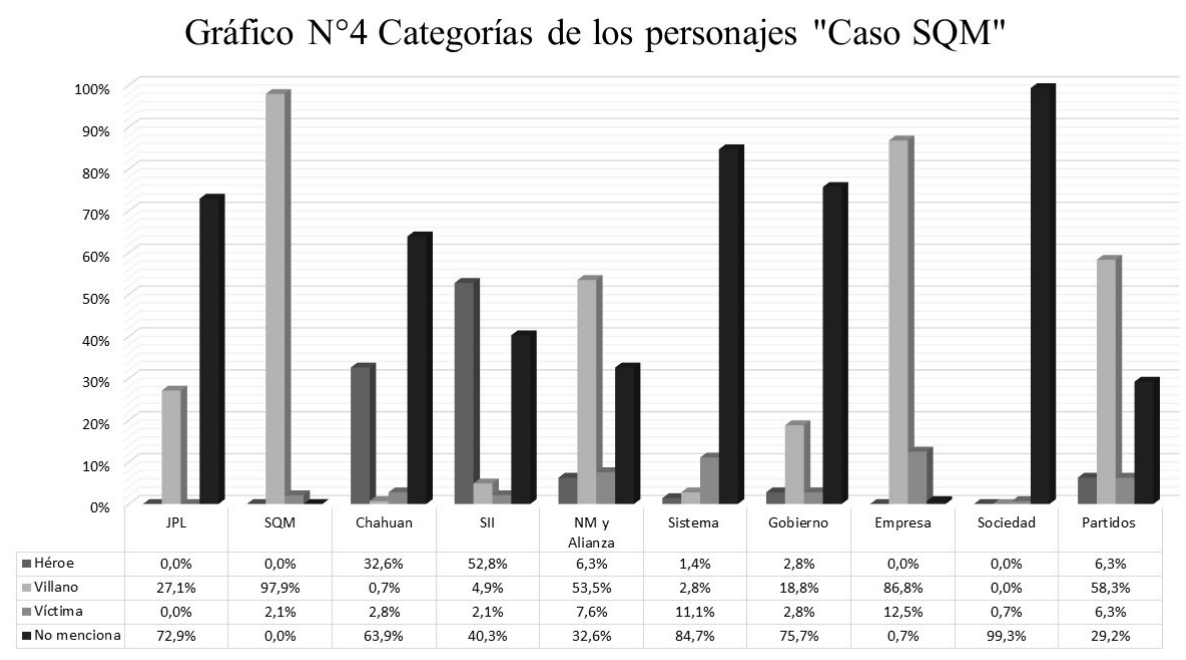

Fuente: Elaboración propia

\subsection{Estrategias discursivas}

Con respecto al tratamiento del discurso y a las intenciones del medio, presentaremos un análisis comparativo entre La Tercera, El Mercurio, El Mundo y El País. En el gráfico $\mathrm{N}^{\circ} 5$ se puede observar como estos medios generaron una visión sobre los casos de corrupción muy particular. En la comparación del "Caso Bárcenas" y el "Caso SQM" se presenta una posición discursiva muy marcada por las omisiones. En lo que respecta al grado de mitigación en el tratamiento noticioso, esta estrategia estuvo presente en el $40,3 \%$ de ítems de la prensa chilena, mientras que en España fue del 34,5\%. La idealización se presentó de manera muy reducida siendo en los periódicos chilenos del 15,3\% y en españoles del 13\%. Con respecto a la omisión está estuvo muy presente dentro de las estrategia periodísticas de ambos países. Del total de 321 noticias analizadas el 62,9\% no buscó mitigar la información sino, por el contrario, consiguió posicionar el tema de la corrupción como un hecho relevante en la agenda. Tampoco se generó un nivel de idealización de los personajes encargados de debelar y juzgar los hechos de corrupción. Sin embargo, gran cantidad de las noticias analizadas se presentaron con omisiones. Esto ocurrió en el $84,4 \%$ del total de ítems analizados, en especial atendiendo a la relación de las empresas con los sistemas de financiamiento de España $(86,4 \%)$ y Chile (81,9\%). 
Gráfico $\mathrm{N}^{\circ} 5$ Estrategias discursivas

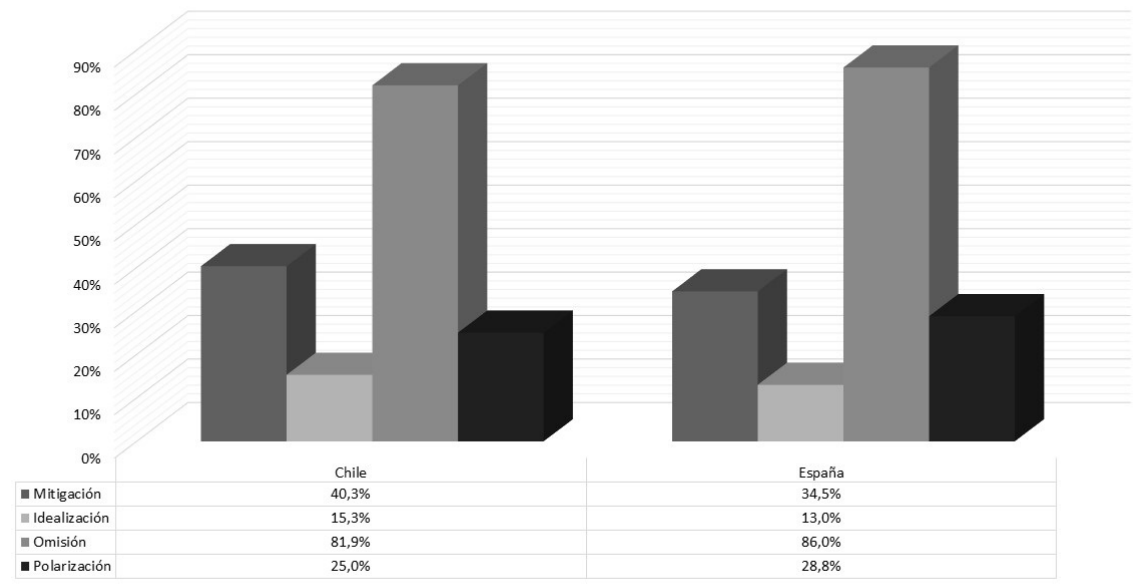

Fuente: Elaboración propia

\section{Discusión}

Los resultados del "Caso Bárcenas" nos permiten identificar que el tratamiento de los periódicos, El Mundo y El País, se focalizó en los personajes, en este caso en Bárcenas y en el PP. Sin embargo, los resultados obtenidos desde las instituciones, las cuales no están relacionadas, dan a entender que la corrupción está contenida en los sujetos corruptos y no en los corruptores. La empresa y el sistema de financiamiento quedan de lado en las relaciones aparecidas en las noticias. Es más, no hay un cuestionamiento de la fuerza gestora de la corrupción en ninguna de las piezas estudiadas. Los poderes políticos, en especial, simbolizados a través del Gobierno y del presidente Mariano Rajoy se llevan todo el peso de la noticia, mientras que se obvian y omiten las raíces o las causas profundas de este fenómeno. El hecho de que no se mencione a las grandes empresas genera una visión incompleta acerca de los sujetos e instituciones involucradas en el caso. Luis Bárcenas, el PP, los partidos y el gobierno son colocados como villanos por la prensa, los cuales se enfrentan al poder de los juzgados. Además, los afectados por la trama de corrupción, la ciudadanía o la sociedad en general, no son mencionadas, ya que se evita realizar la conexión entre los villanos y las víctimas. A este respecto se puede observar que el caso es tratado de manera aislada, ya que los corruptores concretos son simbolizados como los principales generadores de lo que ocurrió, dejando a un lado la raíz de lo que los corrompía.

En el "Caso SQM" la situación cambia, puesto que la empresa se ve directamente involucrada en los relatos ofrecidos por El Mercurio y La Tercera. Julio Ponce Lerou, las empresas y SQM son mencionados como corruptores. Además se les relaciona con los partidos de la Alianza y de la Nueva Mayoría que participaron como corruptores. Sin embargo, dentro del tratamiento de la noticia no se menciona el sistema de financiamiento. Por otra parte, tanto los corruptos y los corruptores son caracterizados como villanos, mientras que el SII y Sabas Chahuán son vistos 
como los héroes. En la relación de villanos y de héroes se da un componente de espectacularización - prácticamente imperceptible en el caso Español. Es más, las noticias chilenas analizadas tienden a dicotomizar la relación entre los "buenos" y los "malos". Esto provoca un nivel de simplificación de la realidad que no permite profundizar en las verdaderas causas del fenómeno. Del mismo modo, la ausencia de la sociedad en las noticias, como víctima primordial de la corrupción, genera una distancia con los lectores, que les impide reconocer las consecuencias negativas de este fenómeno sobre la salud democrática.

En los dos casos de corrupción la omisión de información clave es una estrategia discursiva reiterada. Se tratan de piezas periodísticas en las que lo principal es la confrontación entre héroes y villanos. La omisión de las empresas en el "Caso Bárcenas" es clave, puesto que en este caso los periodistas no mencionan a los corruptores. De esta manera, el poder económico queda oculto, al igual que el sistema de financiamiento o el sistema mismo, que permite con sus leyes que la corrupción se reproduzca y asole la mayoría de las instituciones públicas. El hecho de centrarse en los corruptos como sujetos aislados mitiga el problema en un alto grado.

Para el caso chileno, si bien se acusa a los corruptores (las empresas), existen omisiones en lo que respecta al sistema de financiamiento - que favorece la corrupción- y en la posición de la sociedad. Una diferencia que está presente en el "Caso SQM" es la espectacularización, en la cual los héroes y los villanos son representados por los poderes fiscalizadores y se encuentran ligados a la corrupción. En definitiva, los efectos de la omisión, de la mitigación y de la espectacularización generan una idea de la corrupción muy artificial. Ahora bien, mientras que en el caso español podemos decir que hay elementos de la noticia que provocan que la corrupción se relacione estrictamente con el poder político, dejando de lado a los corruptores y sistema de financiamiento; en el caso chileno el foco está centrado en la investigación que llevan las fuerzas del Estado, dándoles así el rol de velar por los ciudadanos y la democracia.

En ambos casos, la noticia no desarrolla una visión holística de los casos de corrupción, ni favorece un cuestionamiento del sistema mismo por partes de los lectores. El sistema no es culpable ni es responsable, ya que es caracterizado como víctima o está ausente.

\section{Conclusiones}

A la luz del análisis empírico realizado se puede concluir que existen diferencias entre el tratamiento mediático en ambos países. Si bien el caso español permitiría validar la primera hipótesis de este estudio, no ocurre así con el caso chileno. A saber, las noticias españolas sobre el caso Bárcenas se centran en la denuncia de los sujetos corruptos (el extesorero del PP, en este caso), dejando a un lado a los corruptores (el sistema político o los partidos políticos), por ende a los verdaderos responsables del acto de corrupción mismo. Concretamente los ítems estudiados presentan un discurso en el cual los villanos son los únicos culpables. Así, la estrategia planteada por El País y El Mundo es la de obviar la relación entre los círculos empresariales y los partidos políticos. Este podría ser un indicio de la influencia de las grandes empresas en los contenidos desarrollados en los periódicos españoles. Además, la solución que proponen ambos medios, según la disputa entre los personajes es que los villa- 
nos sean juzgados, ya que el problema son "ellos" — los corruptos_-, sin entrar en ningún momento a cuestionar las leyes o el sistema político mismo - corruptorEn contraposición al caso español, las noticias chilenas sobre el caso SQM ofrecen un tratamiento más crítico con la relación corrupto-corruptor, ya que denuncian abiertamente a las empresas como entes corruptores dentro de la trama misma. Valga indicar que en este caso particular, sin embargo, la prensa en Chile no trabajó con el sistema, en general, como personaje abstracto, sino que se centró en los partidos políticos o en las empresas. A este respecto, podríamos afirmar que la primera hipótesis planteada se puede validar en el caso español pero no en el chileno.

En relación a la segunda hipótesis, podríamos validarla de acuerdo a los resultados obtenidos. Ambos países mostraron resultados similares siendo la "omisión" la estrategia discursiva más presente en el tratamiento informativo de ambos casos. La ausencia de datos relevantes, personajes clave o análisis más detallados de las causas provoca que el mensaje que se transmite simplifique las verdaderas causas de la corrupción. Si bien se podría plantear un cuestionamiento de actores políticos y económicos concretos, en ningún caso se hace pensar al lector acerca de las posibles implicancias de la estructura o de las relaciones institucionales y no-institucionales.

En definitiva, la prensa tanto en España como en Chile se ha enfocado sólo en tomar una parte del fenómeno de la corrupción. Esta tendencia pone de manifiesto que existen grandes desafíos para desarrollar un periodismo más especializado en la materia. También preocupa que el nivel de información que está recibiendo la ciudadanía tiene sesgos importantes. El desconectar la relación que existe entre corrupto y corruptor no nos permite identificar ni dimensionar la corrupción. Asimismo, otro problema tiene relación con un análisis más sistémico sobre los casos de corrupción, debido a que siempre son tomados como temas particulares en los que se ha incurrido en malas prácticas. El peligro de mantener la actual posición de la prensa nos llevaría a repetir los mismos errores, puesto que el rol de las comunicaciones sería el generar de límites y percepciones valóricas. El enfoque en personajes concretos canaliza la visión negativa del discurso hacía pequeñas partes del fenómeno de la corrupción. Por el contrario, el dejar de lado la variante sistema perjudica al desarrollo de un periodismo fiscalizador, ya que este actor parece no tener ninguna responsabilidad.

La necesidad de fomentar una posición más crítica en la cobertura de las noticias de corrupción es clave. En el actual contexto, es necesario incentivar un tipo de prensa que sea un aporte en la interpretación de la ciudadanía ante los casos de corrupción. No obstante, tanto en España como en Chile los principales medios han tomado una postura sesgada sobre los contextos en los que se desenvuelven los escándalos políticos y no profundizan ni analizan las verdaderas causas.

\section{Referencias bibliográficas}

Akçay, Selçuk (2006): “Corruption and Human Development”. Cato Journal, 26 (1), 29-45. Washington, D.C., Servicio de publicaciones Cato Institute.

Balán, Manuel (2011): “Competition by Denunciation: The Political Dynamics of Corruption Scandals in Argentina and Chile”. Comparative Politics, 4 (43). Estados Unidos, pp. 459478. DOI: http://dx.doi.org/10.5129/001041511796301597

Barreiro, Belén y Sánchez-Cuenca, Ignacio (2000): "Las consecuencias electorales de la corrupción”. Historia y política: Ideas, procesos y movimientos sociales, 4, 69-92. Madrid. 
Bautista, Oscar (2007): "Marco institucional para combatir la corrupción". Revista Española de Control Externo, 27, 159-197.

Boehm, Frédéric y Graf Lambsdorff, Johann (2009): “Corrupción y anticorrupción: Una perspectiva neo-institucional. Revista de Economía Institucional, 21, 45-74. Colombia.

Caciagli, Mario (1996): "Clientelismo, corrupción y criminalidad organizada". Cuadernos y debates, 60, 156-158. Madrid, Centro de Estudios Constitucionales.

Canel, María José y Sanders, Karen (2005): “El poder de los medios en los escándalos políticos: La fuerza simbólica de la noticia icono". Anàlisi, 32, 163-178.

Canel, María José y Sanders, Karen (2006): Morality Tales: political scandals and journalism in Britain and Spain in the 1990s. Creskill, New Jersey, Hampton Press INC. DOI: 10.1177/1940161208316153.

Castells, Manuel (2003): La Era de la Información: economía, sociedad, cultura y poder. Vol II. España, Siglo XXI.

Centro de Estudios Públicos (2015): “Estudio Nacional de Opinión Pública”. Encuesta CEP, $\mathrm{n}^{\circ} 75$. Chile, pp. 1-80.

Centro de Investigaciones Sociológicas [CIS] (2015): "Barómetro de octubre 2015".

Corzo, Susana (2002): "El clientelismo político como intercambio". Institut de Ciències Politiques i Socials, 206, 3-66. Barcelona.

Dijk, Teun A. van (1999): Ideología: un enfoque multidisciplinario. Barcelona, Gedisa.

Ekaizer, Ernesto (2013): El Caso Bárcenas. España, S.L.U Espasa Libros.

Fontana, Josep (2011): Por el bien del imperio. España, Ediciones de Pasado y Presente.

González, Jesús (1999): "Corrupción, democracia y responsabilidad política". Isonomía: Revista de teoría y filosofía del derecho, 10, 7-24. México.

Gumucio, Rafael (2005): “Chile: corrupción y poder”. POLIS, Revista de la Universidad Bolivariana, 12 (3), 1-16. Chile.

Hierro, José Luis del (2014): "Informe sobre las causas de la corrupción en la sociedad española actual: economía sumergida y comercio ilícito en España”, en Universidad Complutense de Madrid Sitio web:

http://www.thinkcom.es/pdf/informe_causas_corrupcion.pdf [Consulta: 5 de enero de 2016].

Hobsbawm, Eric (1999): Historia del siglo XX. Argentina, Crítica.

Hodgson, Geoffrey y Jiang, Shuxia (2008): “La economía de la corrupción y la corrupción de la economía: una perspectiva institucionalista". Revista de Economía Institucional, 10, 55-80. Colombia.

Kaufmann, Daniel (2000): “Corrupción y reforma institucional: el poder de la evidencia empírica”. Instituto del Banco Mundial, 2 (3), 367-387. Estados Unidos, Washington.

Laguna Platero, Antonio (2013): "La percepción de la corrupción, factor mediatizante de la democracia”. Revista de Ciencias Sociales, 8 (1), 79-98. España.

DOI: 10.14198/OBETS2013.8.1.03.

Larrú, José María (2009): “Corrupción y ayuda al desarrollo evidencias, teoría y aplicaciones para España”. Cuadernos económicos de ICE, 78, 261-280.

Luna, Juan Pablo (2008): "Democracia, gobierno y partidos: Introducción a LAPOP Chile 2008". Revista de Ciencia Política, 3 (28), 115-141. Chile.

DOI:10.1080/01436590802681108.

Martínez, Francesc-Andreu (2013): “Medios de comunicación y escándalos de corrupción en España: ¿denunciantes, magnificadores, cómplices?”. Revista de Ciencias Sociales, 8 (1), 99-126. DOI: 10.14198/obets2013.8.1.04. 
Mönckeberg, María (2002): El saqueo de los grupos económicos al estado chileno. Chile, La nación.

Muñoz, Rafael (2012): “¿Nos importa, realmente, la corrupción?”. Revista de Humanidades, 2 (11), 196-207.

Ordónez, David (2012): “La financiación de los partidos políticos en España: corrupción y deslegitimación". Análisis, 2 (2), 19-26.

Orellana, Patricio (2007): "Chile, un caso de corrupción oculta". Revista de sociología, 21, 257-272. DOI:10.5354/0719-529X.2007.27528.

Pérez Tapias, José (1995): "El homo moralis y su ciudadanía democrática. A propósito de la corrupción: democracia y moral en perspectiva antropológica". Gazeta de Antropología, 11, 1-18.

Pérez, Juan y López, Fernández (2014): “La credibilidad del sistema democrático español: partidos políticos y corrupción”. Revista de Derecho Público, 80, 121-142.

DOI:10.5354/0719-5249.2014.33322.

Qizilbash, Mozaffar (2001): "Corruption and Human Development: A Conceptual Discussion”. Oxford Development Studies, 29 (3), 265-278.

DOI:10.1080/13600810120088868.

Queralt, Joan (2012): “Reflexiones marginales sobre la corrupción”. Revista Crítica Penal y Poder, 2, 18-35.

Rehren, Alfredo (1996): “Corrupción y política local en Chile”. Revista Ciencia Política, 1-2 (18), 141-153. Chile.

Rehren, Alfredo (2000): “Clientelismo político, corrupción y reforma del Estado en Chile”. Centro de Estudios Públicos, Documento de Trabajo n 305, Santiago de Chile, pp. 131163.

Villorria, Manuel y Jiménez, Fernando (2012): “¿Cuánta corrupción hay en España? Los problemas metodológicos de la medición de corrupción (2004-2011)". Revista de Estudios Politicos, 156, 13-47.

Weber, Max (2002): Economía y Sociedad. España, Fondo de Cultura Económica de España.

Zamora, Rocío y Marín, Juan (2010): “La representación simbólica del escándalo de corrupción política. Hacia una tipología de los marcos periodísticos (frames) utilizados en la narración del escándalo de corrupción política”. Razón y Palabra, 73 (15), 1-28. México.

Zamora, Rocío y Marín, Juan (2011): “Un modelo para analizar la representación simbólica del escándalo de corrupción política desde la teoría del framing: aplicación al 'caso Umbra". Miguel Hernández Communication Journal, 2 (12-25), 213-238. España.

Salomé Sola-Morales es Associated Professor en la Escuela de Periodismo de la Universidad de Santiago de Chile. Visiting Professor en la Facultad de Comunicación de la Universidad de Sevilla. Es doctora por la Universidad Autónoma de Barcelona.

Ricardo Rivera Gallardo es Investigador en la Universidad Autónoma de Barcelona. 